\title{
A CASE OF RECURRENCE OF MPNST IN NF 1 WITH INTRACRANIAL AND HEPATIC METASTASES
}

Avijeet Mukherjee, Naveen N, Manohar T. M.

1. Assistant Professor. Department of General Surgery, Adichunchanagiri Institute of Medical Sciences.

2. Assistant Professor. Department of General Surgery, Adichunchanagiri Institute of Medical Sciences.

3. Professor \& HOD. Department of General Surgery, Adichunchanagiri Institute of Medical Sciences.

\section{CORRESPONDING AUTHOR:}

Dr. Naveen. N, \#90, 14, Main, $14^{\text {th }}$ Cross,

$2^{\text {nd }}$ Stage, $2^{\text {nd }}$ Phase,

West of Chord Road,

Mahalakshmipuram, Bangalore - 560086

E-mail: naveen_uno1@yahoo.co.in

Ph: 00919980023372

ABSTRACT: Neurofibromatoses Type 1(NF 1) is an autosomal dominant disease characterized by disordered growth of ectodermal tissues, and is part of a group of disorders called Phakomatoses (neurocutaneous syndrome). NF 1 (aka Von Recklinghausen's disease) patients develop both benign and malignant tumors at an increased frequency. Malignant peripheral nerve sheath tumours (MPNST) is a very rare tumor, with an incidence of 1 per 1,00,000 population. MPNSTs occur in about $2 \%$ to $5 \%$ of neurofibromatosis patients and constitutes between 3 to $10 \%$ of all soft tissue sarcomas. Most MPNSTs are considered as high-grade sarcomas originating from tissues of mesenchymal origin and are biologically aggressive tumours with notoriety for recurrence and metastases.

In this paper, we present a case of a $64 \mathrm{yr}$ old male, who was diagnosed with NF 1 at the age of 25 yrs. He presented with a local recurrence of MPNST in left thigh, He was previously operated for MPNST (Stage 1) at the same site with a tumor free margin, 3 yrs back. Thereafter patient was disease free but two and half yrs later developed a swelling at the same site. Diagnostic core needle biopsy demonstrated a high grade MPNST. Metastatic work up yielded intracranial and hepatic metastases.

KEYWORDS: Neurofibromatosis, Malignant Peripheral Nerve Sheath Tumors, Recurrence, Metastasis

INTRODUCTION: Malignant peripheral nerve sheath tumor (MPNST) is an aggressive and uncommon neoplasm that develops within a peripheral nerve; most cases of which are associated with neurofibromatosis type 1 (NF1). NF1, an autosomal dominant neurocutaneous disorder, is commonly identified by the development of café-au-lait pigmentation of the skin, axillary freckling, optic nerve gliomas, Lisch nodules, distinctive bone lesions, and cutaneous or subcutaneous tumors called neurofibromas ${ }^{1}$. Patients with NF1 develop both benign and malignant tumors at an increased frequency².

MPNST is a rare sarcoma with an incidence of $0.001 \%$ of the general population ${ }^{1}$. It compromises $5 \%-10 \%$ of all soft tissue sarcomas ${ }^{3}$. There is a clear association between MPNST and NF 1, the estimated risk being up to 4600 times greater than in the general population². Although the histogenesis of MPNST remains unclear, there is a higher incidence in patients with prior radiation exposure and neurofibromatosis type 1 (NF1), who have a lifetime risk of 
$10 \%$ of developing MPNST 4,5 . MPNST typically arise in the extremities (40\%), followed by trunk/retroperitoneal (38\%) and head and neck region $(21 \%)^{6}$. Most MPNSTs are biologically high-grade sarcomas that tend to recur (40\%-65\%) and metastasize $(40 \%-80 \%)^{7,8}$.

CASE REPORT: A 64 yr old male patient documented with NF 1 at the age of 25 yrs, presented to the OPD with a rapidly enlarging mass in the left (Lt) thigh which progressively enlarged over a period of 6 months and was associated with pain, paraesthesia and had difficulty in walking. Patient's general condition was poor with refusal for food, headache and altered behaviour.

The patient's clinical history started at the age of 25 years with the complaint of multiple painless swellings all over the body. The patient was diagnosed with NF 1 . Three yrs back at the age of 61 yrs patient had presented in our OPD with a swelling at the same site wherein a neurofibromatous swelling rapidly increased in size associated with pain. Core biopsy of the swelling yielded a low grade MPNST. Physical examination revealed multiple neurofibromas (including plexiform variety) of the chest and trunk, multiple café-au-lait spots of the axillary and gluteal areas and trunk, scoliosis and a Lisch nodule of the right eye. X-ray of the Lt lower limb showed a homogenous mass with no bone involvement. Metastatic workup was done and none found. The patient was staged according to AJCC system for soft tissue sarcomas as Stage 1 and tumor was surgically resected with a macroscopic tumor free margin of $3 \mathrm{cms}$ all around. HPE revealed a microscopic tumor free margin of $2 \mathrm{cms}$. Patient was discharged thereafter and followed up for 6 months, after which patient was lost for follow up.

Two and a half years later patient noticed a swelling of about $1 \times 2 \mathrm{cms}$ in size at the operated site which gradually increased to the present size of $8 \times 5 \mathrm{cms}$ and was associated with pain and paraesthesia of the Lt lower limb. On general physical examination, multiple neurofibromas (including plexiform variety) of the face, chest, trunk and extremities with multiple and diffuse café-au-lait spots of the axillary and gluteal areas and trunk (around 6 spots were determined to be larger than $1.5 \square \mathrm{cm}$ ), and scoliosis [Fig 1\&2]. Ophthalmologic examination revealed Lisch nodule present in both eyes. On local examination, a swelling measuring $8 \times 5 \mathrm{cms}$ was present over the anterior part of Lt thigh with central ulceration and hyperpigmentation of the surrounding area. The swelling was tender with induration. Ulcer was $3 \times 2 \mathrm{cms}$ and oval in shape with sloping edges and floor covered with slough [Fig 3]. Lt inguinal lymph nodes were palpable 6 in number, largest measuring $2 \times 1 \mathrm{cms}$, tender, firm in consistency and mobile. Per abdomen examination revealed hepatomegaly with bosselated surface and moderate ascites. Respiratory examination showed bilateral crepitations. Cardiovascular examination was normal. On CNS examination, patient alertness was low associated with lethargy.

Diagnostic core needle biopsy of the tumor on microscopic examination showed spindle cell with fascicular pattern and varying degrees of mitosis, necrosis and calcification with presence of associated benign neurofibroma or schwannanian cells, and positive immunohistochemical staining for S-100 protein [Fig 4]. HPE of Lt inguinal nodes showed no evidence of nodal metastases. X-Ray of Lt lower limb showed swelling in the thigh with scattered calcification and femur was normal. CT abdomen showed metastases in liver. MRI brain showed contrast-enhancing lesion in the Rt frontal lobe [Fig 5].

Based on the patient's poor prognosis due to extensive tumor spread in terms of metastases to liver and distant brain and poor general condition of the patient, removal of the recurrent tumor in the thigh was thought to be unjustified. So, palliative radiochemotherapy was thought to be of a better approach in view of poor prognosis. The same was conveyed to the 
patient's family and to which they were reluctant for any further management. Patient was symptomatically treated but within a week patient succumbed.

DISCUSSION: Neurofibromas, most common benign tumors in NF 1, are composed of an admixture of Schwann cells, perineural fibroblasts, and mast cells infiltrating the peripheral nerves $^{1,2}$. MPNSTs may develop within an associated neurofibroma ${ }^{2,9}$. The malignant transformation of a neurofibroma has an extremely poor prognosis with prevalent recurrences and distant metastasis 9 , 10 .

For the clinical challenge to diagnose MPNST, the pathologist has to deal with a great work up to provide a histological diagnosis of MPNST and requires careful differentiation from a number of tumor entities with similar histologic features. An immunoreaction to S-100 as in our case excludes a fibrosarcoma and underlines the close association of MPNST with the nerve sheaths ${ }^{11,12}$.

The diagnosis of a recurrent MPNST with distant metastases (in particular, if the tumor has spread into the Central Nervous System) is a clinical challenge for the surgeon regarding treatment options and poor outcome of the current treatment modalities. No uniform criteria have been established for the treatment of recurrent MPNST with widespread metastases. However, it is widely agreed that the extent of surgical resection is an important prognostic factor while no definitive data is available on the benefit of postoperative radiotherapy ${ }^{13}$. Irradiation seems to reduce local recurrence but does not affect the overall survival rate. Evidence for any relevant cytotoxic effect of chemotherapy (doxorubicin, ifosfamide) is not available ${ }^{14}$ and is only palliative.

In most published cases of MPNST metastasizing to the cerebral parenchyma, the metastasis was removed microsurgically. Whole-brain irradiation without prior surgical removal or a radiosurgical approach in treating cerebral metastasis of a MPNST is the best choice.

MPNST in general has an unfavorable prognosis with a 5-year survival rate of only $64 \%$. Survival is even much shorter in patients with a brain metastasis and is not affected by the therapeutic approach. A definitive appraisal of the situation is difficult because only few patients have been treated so far. Our treatment plan for a palliative radiochemotherapy in the above case was thought to be the best approach in view of the recurrence of the primary tumor (in spite of achieving a tumor free margin of $2 \mathrm{cms}$ at first instance), distant metastases (brain and liver), poor general condition of the patient and the financial burden on the family. In our hospital, where we cater the rural population, it's difficult to elucidate the patients and their family about the disease (NF 1, MPNST), unavailability of any definitive treatment strategy, poor follow up thereafter and most importantly financial constraints. In the current scenario, our approach to such patients has only been to relieve the patients of their symptoms rather than a curative approach in view of the poor survival rate.

\section{REFERENCES:}

1. Neurofibromatosis. Conference statement. National Institutes of Health Consensus Development Conference: Archives of Neurology 1988, vol. 45, pp. 575-578.

2. R. E. Ferner and D. H. Gutmann, International consensus statement on malignant peripheral nerve sheath tumors in neurofibromatosis: Cancer Research 2002, vol. 62, no. 5, pp. 1573-1577. 
3. Weiss SW, Goldblum JR. St Louis, MO: Mosby, Inc.; 2007. Enzinger and Weiss's Soft Tissue Tumors.

4. Evans DG, Baser ME, McGaughran J, et al. Malignant peripheral nerve sheath tumours in neurofibromatosis. J Med Genet 2002, 39:311-314.

5. Ducatman BS, Scheithauer BW, Piepgras DG, et al. Malignant peripheral nerve sheath tumors: A clinicopathologic study of 120 cases. Cancer 1986, 57:2006-2021.

6. Carli M, Ferrari A, Mattke A, et al. Pediatric malignant peripheral nerve sheath tumor: the Italian and German soft tissue sarcoma cooperative group. J Clin Oncol 2005, 23:8422-8430.

7. Anghileri M, Miceli R, Fiore M, et al. Malignant peripheral nerve sheath tumors: prognostic factors and survival in a series of patients treated at a single institution. Cancer 2006, 107:1065-1074.

8. Wong WW, Hirose T, Scheithauer BW, et al. Malignant peripheral nerve sheath tumor: analysis of treatment outcome. Int J Radiat Oncol Biol Phys 1998, 42:351-360.

9. O. A. Sheikh, A. Reaves, F. A. Kralick, et al. Malignant nerve sheath tumor of the spinal accessory nerve: a unique presentation of a rare tumor. Journal of Clinical Neurology 2012, vol. 8, pp. 75-78.

10. E. A. Munhoz, C. L. Cardoso, E. S. Tolentino, et al. Von Recklinghausen's diseasediagnosis from oral lesion. Neurofibromatosis. International Journal of Odontostomatology 2010, vol. 4, pp. 179-183.

11. Matyja E, Naganska E, Gorski R, Zabek M. Multiple brain metastases from malignant peripheral nerve sheath tumour (MPNST). Folia Neuropathol 2004, 42:43-48.

12. Kuschner M, Laskin S. Experimental models in environmental carcinogenesis. Am J Pathol 1971, 64:183-196.

13. Perrin RG, Guha A: Malignant peripheral nerve sheath tumors. Neurosurg Clin N Am 2004, 15:203-216.

14. Adjuvant chemotherapy for localised resectable soft-tissue sarcoma of adults: metaanalysis of individual data. Sarcoma Meta-analysis Collaboration Lancet 1997, 350:1647-1654. 


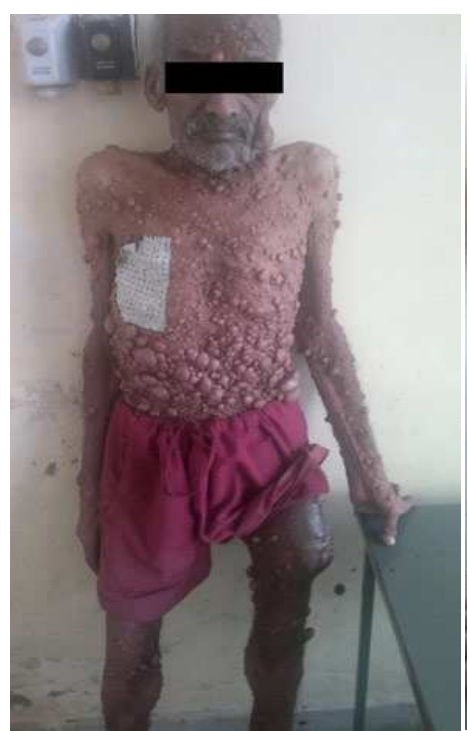

Fig 1 - Neurofibromas seen on face, trunk and extremities with scoliosis (Note the patient supporting himself With his left hand on the table)

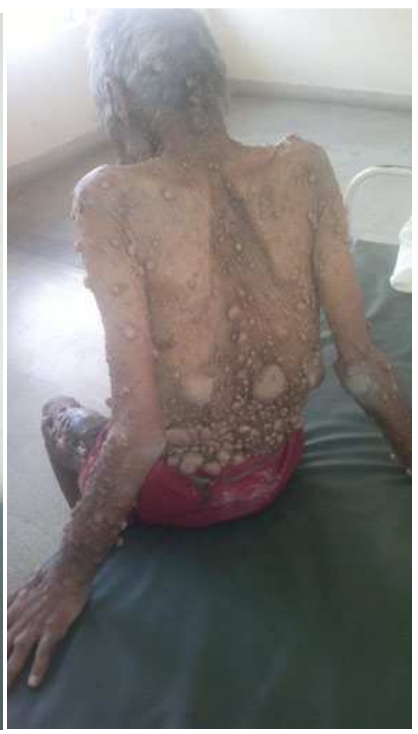

Fig 2 - Multiple neurofibromatous swellings with plexiform variety over the back
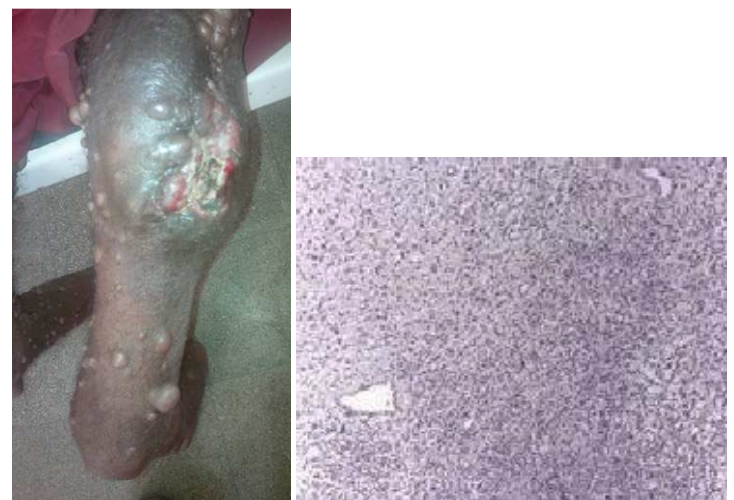

Fig 3 - Recurrent tumor (MPNST) in left thigh Fig 4 - Microscopic examination of the tumor showing Schwann cells, perineural fibroblasts, and mast cells with varying degrees of mitosis, necrosis and calcification

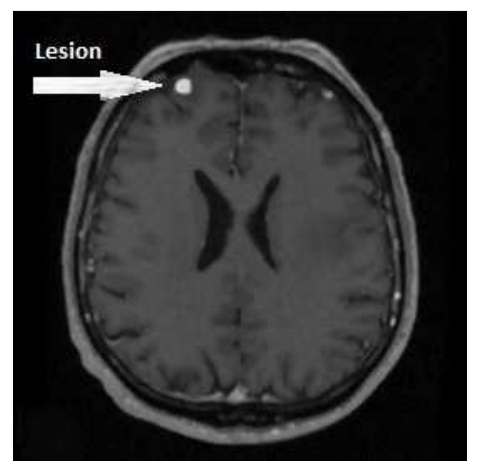

Fig 5 - MRI head showing contrast enhancing intracranial metastatic lesion in right frontal lobe 